The Journal of the American Osteopathic Association August/1983

\title{
Trends in cesarean section rate
}

Use of butorphanol tartrate in balanced anesthesia

Rotavirus outbreak in a newborn nursery

CME quiz, page 13

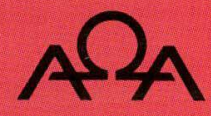

BCDEFGHIJKLMNOPQRSTUVWXYZ\&AB

DEFGHIJKLMNOPQRSTUVWXYZ\&ABCD IFGHIJKLMNOPQRSTUVWXYZ・ANNUAL NDEX • VOLUME 82 • SEPTEMBER 1982 UGUST 1983 • ABCDEFGHIJKLMNOPQRS UVWXYZ\&ABCDEFGHIJKLMNOPQRSTU WXYZ \& ABCDEFGHIJKLMNOPQRSTUV 


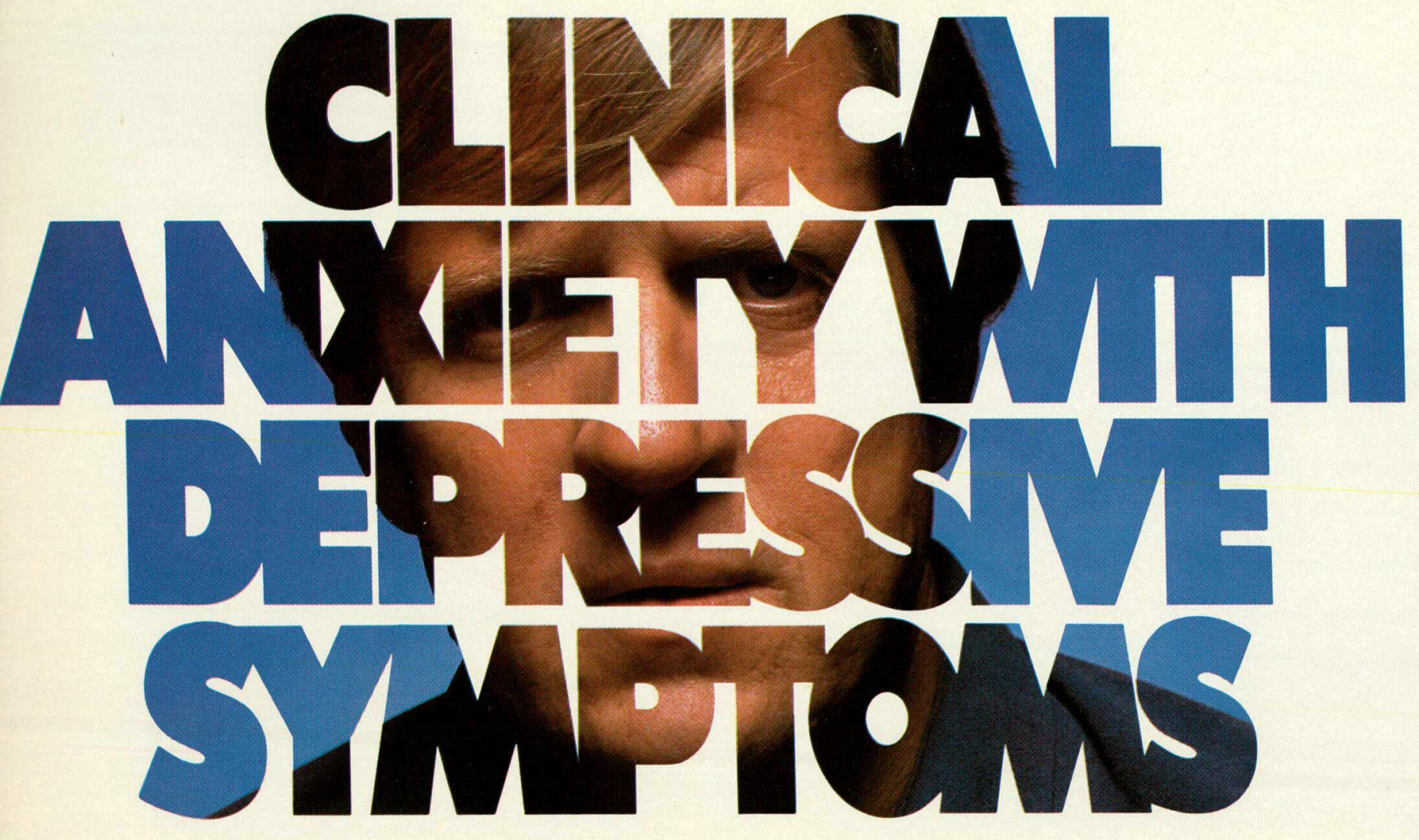

In a controlled clinical study of 1,168 patients diagnosed as having moderate to severe anxiety, over $70 \%$ also exhibited a moderate to severe depressed mood as elicited on the Hamilton Anxiety Rating Scale (HARS).*

On the Self-Rating Symptom Scale, a large number of patients also reported experiencing such depressive symptoms as low energy, feeling blue and lonely, self-blame, and difficulty making decisions** 